ONDERZOEKSRAPPORT NR 9427

\title{
On Approximating Distributions by Approximating their De Pril Transforms
}

by

Jan DHAENE

Bjørn SUNDT

D/1994/2376/29 


\title{
On approximating distributions by approximating their De Pril transforms
}

\author{
by \\ Jan Dhaene, Katholieke Universiteit Leuven \\ and \\ Bjørn Sundt, The Wyatt Company, Oslo
}

\begin{abstract}
Dhaene \& De Pril (1994) gave some general bounds for errors caused by applying an approximation to the De Pril transform of a probability distribution. In this paper we further analyse such bounds. We prove results for several simple situations and indicate how these results can be combined for more complex situations. As an illustration we apply the results to deduce approximations to the convolution of a finite number of compound distributions whose counting distributions belong to a class consisting of the binomial, Poisson, and negative binomial distributions, and we give error bounds for these approximations.
\end{abstract}

Keywords: Approximations, error bounds, De Pril transforms.

Abbreviated title: Approximating De Pril transforms.

Leuven/Oslo, November 7, 1994. 


\section{Introduction}

Let $\mathcal{P}_{0}$ denote the class of discrete probability densities on the non-negative integers with a positive probability in zero. We define the De Pril transform $\varphi_{f}$ of $f \in \mathcal{P}_{0}$ by the recursion

$\varphi_{f}(x)=\frac{1}{f(0)}\left[x f(x)-\Sigma_{y=1}^{x-1} \varphi_{f}(y) f(x-y)\right] ; \quad(x=0,1, \ldots)$

in this paper we shall interpret $\Sigma_{i=a}^{b} v_{i}=0$ and $\Pi_{i=a}^{b} v_{i}=1$ when $a>b$.

By solving (1.1) with respect to $f(x)$ we obtain

$f(x)=\frac{1}{x} \Sigma_{y=1}^{\mathcal{x}} \varphi_{f}(y) f(x-y) . \quad(x=1,2, \ldots)$

Conversely, we obtain (1.1) by solving (1.2) with respect to $\varphi_{f}(x)$. Thus we see that when we know $\varphi_{f}$ and $f(0)$, then we can evaluate $f$ recursively by (1.2). On the other hand, if $f$ is known, then we can evaluate $\varphi_{f}$ recursively by (1.1).

As we need the value of $f(0)$ to start the recursion (1.2), one might get the impression that $f$ is not uniquely determined by $\varphi_{f}$. However, the uniqueness follows from the fact that $f$ is the discrete density of a probability distribution, which implies that

$\Sigma_{x=0}^{\infty} f(x)=1$

Unfortunately, this condition is usually not convenient for starting the recursion (1.2), and we therefore rather use the value of $f(0)$ which would normally be known.

The following theorem, which was first proved by De Pril (1989), indicates 
the usefulness of De Pril transforms and the recursions (1.1) and (1.2).

Theorem 1.1. The De Pril transform of the convolution of a finite number of discrete densities in $\mathcal{P}$ is the sum of the De Pril transforms of these discrete densities.

From Theorem 1.1 we see that we can evaluate the convolution of a finite number of discrete densities in $\mathcal{P}_{0}$ by first finding the De Pril transform of each of the densities, e.g. by using the recursion (1.1), then finding the De Pril transform of the convolution by using Theorem 1.1, and finally finding the discrete density of the convolution recursively by (1.2).

Unfortunately, this procedure can be rather time-consuming. Therefore De Pril (1989) suggested an approximative algorithm that can be applied with less resources, and he gave upper bounds for the approximation error. He also gave similar bounds for other approximations to convolutions, introduced by Kornya (1983) and Hipp (1986). The presentation of these approximations and the deduction of their error bounds were unified by Dhaene \& De Pril (1993). They proved a general result, showing how one from an error bound for the approximation to the De Pril transform can deduce error bounds for the approximation to the discrete density, and they gave similar results for approximations to the cumulative distribution and the stop loss transform.

In the present paper we shall discuss some consequences of the general results of Dhaene \& De Pril (1994). We shall approximate probability distributions on the non-negative integers by approximating their discrete densities. Thus we identify a distribution by its discrete density, and for convenience we shall therefore mean its discrete density when talking about a distribution.

As the approximations to the distributions are not necessarily distributions 
themselves, we shall extend the notion of De Pril transforms to more general functions. This is the topic of Section 2. In Section 3 we discuss the main results of Dhaene \& De Pril (1994). Section 4 is devoted to the classes $\mathcal{R}_{k}$ studied by Sundt (1992). In Sections 5, 6, and 7 we present results on approximations to respectively convolutions, compound distributions, and infinitely divisible distributions. Some of the results in Sections 4-7 may seem trivial and unnecessary as they concern approximations in situations where we can apply exact methods without too much effort. However, in the final Section 8 we shall show how these simple elements can be applied as building blocks in more complex situations where exact methods would be very time-consuming. As an illustration, we apply the results to deduce approximations to the convolution of a finite number of compound distributions whose counting distributions belong to a class consisting of the binomial, Poisson, and negative binomial distributions, and we give error bounds for these approximations. De Pril's (1989) approximation appears as a special case by letting the counting distributions be Bernoulli distributions.

\section{De Pril transforms of functions}

$2 \mathrm{~A}$. Let $\mathcal{F}_{0}$ denote the class of all functions $f$ on the non-negative integers with $f(0)>0$. Then $\mathcal{P}_{0} \subset \mathcal{F}_{0}$. We extend the definition (1.1) of De Pril transforms to functions $f \in \mathcal{F}_{0}$. We also introduce the classes $\mathcal{F}$ and $\mathcal{F}_{+}$of respectively all functions on the non-negative integers and all functions on the positive integers and the corresponding classes $\mathcal{P}$ and $\mathcal{P}_{+}$restricted to (discrete densities of) probability distributions.

We see that also for functions $f \in \mathcal{F}_{0}$, we can evaluate $f$ recursively by (1.2) if we know $\varphi_{f}$ and $f(0)$. However, as we do not any longer have the constraint (1.3), 
the function $f$ is no longer uniquely determined by $\varphi_{f}$; it is only determined up to a multiplicative constant, that is, the set of all functions with De Pril transform $\varphi_{f}$ is the set of functions $c f$ with a positive constant $c$.

2B. Instead of expressing their results in terms of De Pril transforms, Dhaene $\&$ De Pril (1994) represented a function $f \in \mathcal{F}_{0}$ by the transform $\psi_{f}$ given by

$\psi_{f}(0)=\ln f(0)$

$\psi_{f}(x)=\frac{\varphi_{f}(x)}{x}$.

$$
(x=1,2, \ldots)
$$

Using the transform $\psi_{f}$ instead of $\varphi_{f}$ has the advantage that the term $\psi_{f}(0)$ ensures that $f$ is uniquely determined by $\psi_{f}$, and some deductions look more tidy in terms of this representation. On the other hand, recursions like (1.1) and (1.2) look simpler in terms of De Pril transforms, and the use of this transform fits more naturally into the framework discussed by Sundt (1992), to which we shall return in Section 4.

2C. Let $\rho_{f}$ be the generating function of $f$, that is,

$\rho_{f}(s)=\Sigma_{x=0}^{\infty} f(x) s^{x}$

If $f \in \mathcal{P}_{0}$, then $\rho_{f}$ is the probability generating function of the corresponding distribution. The transforms $\varphi_{f}$ and $\psi_{f}$ can be defined by the power series expansions

$$
\Psi_{f}(s)=\ln \rho_{f}(s)=\Sigma_{x=0}^{\infty} \psi_{f}(x) s^{x} \quad \Phi_{f}(s)=s \frac{\mathrm{d}}{\mathrm{d} s} \ln \rho_{f}(s)=\Sigma_{x=1}^{\infty} \varphi_{f}(x) s^{x},
$$


provided that these series have positive radiuses of convergence. This is always the case when $f \in \mathcal{P}_{0}$.

\section{Some general results on error bounds}

3A. In this section we shall discuss some results from Dhaene \& De Pril (1994).

For $f, g \in \mathcal{F}_{0}$ we define

$\delta_{j}(f, g)=\Sigma_{x=1}^{\infty} x^{j-1}\left|\varphi_{f}(x)-\varphi_{g}(x)\right|$
$\delta(f, g)=\delta_{0}(f, g)+\left|\ln \frac{f(0)}{g(0)}\right|$

and, provided that these series converge,

$\nu_{j}(g)=\Sigma_{x=1}^{\infty} x^{j-1} \varphi_{g}(x)$

We also introduce

$\mu_{j}(h)=\Sigma_{x=0}^{\infty} x^{j} h(x)$

for functions $h \in \mathcal{F}$ for which these series converge. If $h \in \mathcal{P}$, then $\mu_{0}(h)=1$ and $\mu_{1}(h)$ is the mean of $h$. When we apply $\nu_{j}(g)$ and $\mu_{j}(g)$ in the sequel, we shall always silently assume that the associated series converge, that is, the results involving $\nu_{j}(g)$ and $\mu_{j}(g)$ are valid when the associated series converge.

We shall also need the quantities 
$\epsilon_{j}(h, k)=\Sigma_{x=0}^{\infty} x^{j}|h(x)-k(x)| . \quad(j=0,1 ; h, k \in \mathcal{F})$

Theorem 3.1. For $f \in \mathcal{P}_{0}$ and $g \in \mathcal{F}_{0}$ we have

$\epsilon_{0}(f, g) \leq e^{\delta(f, g)}-1$

For $f \in \mathcal{F}$ we define

$\Gamma_{f}(x)=\Sigma \underset{y=0}{x} f(y)$

$(x=0,1, \ldots)$

If $f \in \mathcal{P}$, then $\Gamma_{f}$ is the corresponding cumulative distribution.

Corollary 3.1. For $f \in \mathcal{P}_{0}$ and $g \in \mathcal{F}_{0}$ we have

$$
\left|\Gamma_{f}(x)-\Gamma_{g}(x)\right| \leq\left(e^{\delta(f, g)}-1\right) \Gamma_{f}(x) \leq e^{\delta(f, g)}-1 . \quad(x=0,1, \ldots)
$$

Corollary 3.2. For $f \in \mathcal{P}_{0}, g \in \mathcal{F}_{0}$, and $A \subset\{0,1, \ldots\}$ we have

$$
\left|\Sigma_{x \in A} f(x)-\Sigma_{x \in A} g(x)\right| \leq \frac{1}{2}\left[e^{\delta(f, g)}-1+\left|1-\mu_{0}(g)\right|\right] \leq e^{\delta(f, g)}-1 .
$$

The stop loss transform of the cumulative distribution $\Gamma_{f}$ with $f \in \mathcal{P}$ is defined by

$$
\Pi_{f}(x)=\Sigma \Sigma_{y=x+1}^{\infty}(y-x) f(y), \quad(x=0,1, \ldots)
$$

and it is natural to extend this definition to functions $f \in \mathcal{F}$. Thus, if we approximate $f \in \mathcal{P}$ with $g \in \mathcal{F}$, we can approximate $\Pi_{f}$ by $\Pi_{g}$ (provided that $\mu_{1}(f), \mu_{0}(g)$ and 
$\mu_{1}(g)$ converge). We introduce

$\eta(p, q)=\sup _{x \geq 0}\left|\Pi_{p}(x)-\Pi_{q}(x)\right|$

$(p, q \in \mathcal{F})$

Alternatively we can write $\Pi_{g}(x)$ as

$\Pi_{g}(x)=\Sigma_{y=0}^{x-1}(x-y) g(y)+\mu_{1}(g)-x \mu_{0}(g) . \quad(x=0,1, \ldots)$

As $f \in \mathcal{P}$, we obtain in particular

$\Pi_{f}(x)=\Sigma_{y=0}^{x-1}(x-y) f(y)+\mu_{1}(f)-x . \quad(x=0,1, \ldots)$

We have that $\mu_{1}(f)$ is the mean of the distribution, and in practice this could usually be easily found. Thus it is also natural to approximate $\Pi_{f}$ by $\Pi_{g}^{(f)}$ given by

$$
\Pi_{g}^{(f)}(x)=\Sigma_{y=0}^{x-1}(x-y) g(y)+\mu_{1}(f)-x . \quad(x=0,1, \ldots)
$$

Not surprisingly, the error bounds presented by Dhaene \& De Pril (1994) indicate that $\Pi_{g}^{(f)}(x)$ gives a better approximation than $\Pi_{g}(x)$ for low values of $x$ whereas $\Pi g(x)$ is best for high values of $x$.

Theorem 3.2. If $f \in \mathcal{P}_{0}$ and $g \in \mathcal{F}_{0}$, then

$$
\begin{aligned}
& \left|\Pi_{f}(x)-\Pi_{g}(x)\right| \leq\left[e^{\delta(f, g)}-1\right] \Pi_{f}(x)+\delta_{1}(f, g) e^{\delta(f, g)} \quad(x=0,1, \ldots) \\
& \eta(f, g) \leq\left[e^{\delta(f, g)}-1\right] \mu_{1}(f)+\delta_{1}(f, g) e^{\delta(f, g)}
\end{aligned}
$$


Theorem 3.3. For $f \in \mathcal{P}_{0}$ and $g \in \mathcal{F}_{0}$, we have

$$
\left|\Pi_{f}(x)-\Pi_{g}^{(f)}(x)\right| \leq\left(e^{\delta(f, g)}-1\right)\left[\Pi_{f}(x)+x-\mu_{1}(f)\right] . \quad(x=0,1, \ldots)
$$

We see that the first upper bound in Corollary 3.1 depends on $\Gamma_{f}(x)$, which will normally be unknown. Analogously, the bounds in Theorems 3.2 and 3.3 depend on $\Pi_{f}(x)$, which will normally be unknown. From the following lemma we can construct computable bounds for these situations.

Lemma 3.1. If $0 \leq c<1$ and $|a-b| \leq c a+d$, then

$$
|a-b| \leq \frac{c b+d}{1-c}
$$

Proof. We have

$$
\begin{aligned}
& |a-b| \leq c(|a-b|+b)+d \\
& |a-b|(1-c) \leq c b+d
\end{aligned}
$$

from which we obtain (3.4).

Q.E.D.

By application of Lemma 3.1 to the inequalities in Corollary 3.1 and Theorems 3.2 and 3.3, we obtain the following bounds, which can also be found in Dhaene \& De Pril (1994).

Corollary 3.3. For $f \in \mathcal{P}_{0}$ and $g \in \mathcal{F}_{0}$ with $\delta(f, g)<\ln 2$ and $x=0,1, \ldots$ we have the following bounds 


$$
\begin{aligned}
& \left|\Gamma_{f}(x)-\Gamma_{g}(x)\right| \leq \frac{e^{\delta(f, g)}-1}{2-e^{\delta(f, g)} \Gamma_{g}(x)} \\
& \left|\Pi_{f}(x)-\Pi_{g}(x)\right| \leq \frac{\left(e^{\delta(f, g)}-1\right) \Pi_{g}(x)+\delta_{1}(f, g) e^{\delta(f, g)}}{2-e^{\delta(f, g)}} \\
& \eta(f, g) \leq \frac{\left(e^{\delta(f, g)}-1\right) \mu_{1}(g)+\delta_{1}(f, g) e^{\delta(f, g)}}{2-e^{\delta(f, g)}} \\
& \left|\Pi_{f}(x)-\Pi_{g}^{(f)}(x)\right| \leq \frac{e^{\delta(f, g)}-1}{2-e^{\delta(f, g)}}\left(\Pi_{g}^{(f)}(x)+x-\mu_{1}(f)\right) .
\end{aligned}
$$

The following result was also proved by Dhaene \& De Pril (1994).

Theorem 3.4. If $g \in \mathcal{F}_{0}$ such that $\Sigma_{x=1}^{\infty}\left|\varphi_{g}(x)\right|<\infty$, then

$$
\mu_{0}(g)=g(0) e^{\nu_{0}(g)} \quad \mu_{1}(g)=\mu_{0}(g) \nu_{1}(g)
$$

3B. We see that in all the situations presented in the previous subsection, the upper bounds for the approximation errors were increasing functions of $\delta_{0}(f, g)$, as well as $\delta_{1}(f, g)$, to the extent that this latter quantity appears in the bounds. Thus we can replace these quantities by larger quantities in the upper bounds. In the following sections we shall therefore deduce upper bounds for $\delta_{0}(f, g)$ and $\delta_{1}(f, g)$ in various situations.

3C. The upper bounds in Theorems $3.1-3$ and Corollary 3.1 depend on $g$ only through $\delta(f, g)$ and $\delta_{1}(f, g)$. Furthermore, $\delta_{0}(f, g)$ and $\delta_{1}(f, g)$ depend on $g$ only through $\varphi_{g}$. As $\varphi_{c g}=\varphi_{g}$ for any positive constant $c$, we have

$$
\delta(f, \mathrm{c} g)=\delta_{0}(f, g)+\left|\ln \frac{f(0)}{c g(0)}\right| \quad \delta_{1}(f, c g)=\delta_{1}(f, g)
$$


This implies that the upper bounds mentioned above are minimised by choosing $c=f(0) / g(0)$. In other words, we improve the upper bounds by using the starting value $f(0)$ in the recursion (1.2) for the approximation.

This result seems to indicate a deficiency of the upper bounds in subsection 3A. For example, if $g(0)=f(0)$ and $g(x)$ is much smaller than $f(x)$ for all $x>0$, then we could improve our approximation by multiplying $g$ by some constant greater than one, but that would increase our upper bounds for the approximation errors.

In particular, Kornya's (1983) approximation is equal to De Pril's (1989) approximation up to to a multiplicative constant. As De Pril's approximation is exact in zero, this approximation obtains the lowest upper bounds.

\section{The classes $\mathcal{R}_{k}$}

4A. Sundt (1992) denoted by $R_{k}[\mathbf{a}, \mathbf{b}]$ the distribution with discrete density $f \in \mathcal{P}_{0}$ defined by the recursion

$f(x)=\Sigma_{y=1}^{k}\left[a_{y}+\frac{b y}{x}\right] f(x-y) \quad(x=1,2, \ldots)$

with $\mathbf{a}=\left(a_{1}, \ldots, a_{k}\right), \mathbf{b}=\left(b_{1}, \ldots, b_{k}\right)$, and $f(x)=0$ for all $x<0$. By $\mathcal{R}_{k}$ he denoted the class of all such distributions with fixed $k$ and by $\mathcal{R}_{k}^{0}$ the class

$\mathcal{R}_{k}^{0}=\mathcal{R}_{k} \sim \mathcal{R}_{k-1}$

of all distributions in $\boldsymbol{R}_{k}$ that cannot be represented by a lower order recursion in the form (4.1). In particular, we see that $\mathcal{R}_{\infty}=\mathcal{P}_{0}$, and from (1.2) we see that in 
terms of De Pril transform, the distribution $f \in \mathcal{P}_{0}$ is $\mathbb{R}_{\infty}[0, c]$ with $c_{i}=\varphi_{f}(i)$ $(i=1,2, \ldots)$.

For approximating the distribution $R_{k}[\mathbf{a}, \mathbf{b}]$, we want to apply a function $g \in \mathcal{F}_{0}$ which also satisfies a recursion in the form (4.1). We shall say that a function in $\mathcal{F}_{0}$ is in the form $R_{k}[\mathrm{a}, \mathrm{b}]$ if it satisfies the recursion (4.1). Whereas a distribution $R_{k}[\mathrm{a}, \mathrm{b}]$ is uniquely determined by $\mathbf{a}$ and $\mathbf{b}$, this is not the case with a function in the form $R_{k}[\mathbf{a}, \mathbf{b}]$; like with the De Pril transform it is only determined up to a multiplicative constant.

The "if" part of the following theorem was proved by Sundt (1993) in the special case $f \in \mathcal{P}_{0}$.

Theorem 4.1. A function $f \in \mathcal{F}_{0}$ is in the form $R_{k}[\mathrm{a}, \mathrm{b}]$ if and only if its De Pril transform satisfies the recursion

$\varphi_{f}(x)=x a_{x}+b_{x}+\Sigma_{y=1}^{k} a_{y} \varphi_{f}(x-y) \quad(x=1,2, \ldots)$

with $a_{x}=b_{x}=0$ for $x>k$ and $\varphi_{f}(x)=0$ for $x<0$.

Proof. Let $f \in \mathcal{F}_{0}$ be in the form $R_{k}[\mathrm{a}, \mathrm{b}]$. We shall prove (4.2) by induction.

For $x=1$, we obtain from (1.1)

$\varphi_{f}(1)=\frac{f(1)}{f(0)}=a_{1}+b_{1}$

that is, (4.2) holds for $x=1$.

Let us now assume that (4.2) holds for $x=1,2, \ldots, v-1$. We shall show that then (4.2) also holds for $x=v$. Insertion of (4.1) and (4.2) in (1.1) gives 
$\varphi_{f}(v)=\frac{1}{f(0)}\left[v f(v)-\Sigma_{y=1}^{v-1} \varphi_{f}(y) f(v-y)\right]=$

$\frac{1}{f(0)}\left[\Sigma_{y=1}^{v}\left(v a_{y}+b_{y}\right) f(v-y)-\Sigma_{y=1}^{v-1}\left[y a_{y}+b_{y}+\Sigma_{z=1}^{k} a_{z} \varphi_{f}(y-z)\right] f(v-y)\right]=$

$v a_{v}+b_{v}+\frac{1}{f(0)} \Sigma_{z=1}^{v-1} a_{z}\left[(v-z) f(v-z)-\Sigma_{y=1}^{v-1} \varphi_{f}(y-z) f(v-y)\right]=$

$v a_{v}+b_{v}+\Sigma_{z=1}^{k} a_{z} \varphi_{f}(v-z)$

that is, (4.2) holds for $x=v$.

By induction it follows that (4.2) holds for all $x$.

The converse implication follows from the fact that a function is determined by its De Pril transform up to a multiplicative constant, and thus Theorem 4.1 is proved.

Q.E.D.

Theorem 4.2. If $f \in \mathcal{P}_{0}$ is in the form $R_{k}[\mathbf{a}, \mathbf{b}]$ with $\Sigma_{i=1}^{k}\left|a_{i}\right|<1$, and $g \in \mathcal{F}_{0}$ is in the form $R_{k}[\mathbf{c}, \mathrm{d}]$, then

$\delta_{j}(f, g) \leq \frac{\Sigma_{x=1}^{k} x^{j-1}\left|x\left(a_{x}-c_{x}\right)+b_{x}-d_{x}\right|+\left[\Sigma_{x=1}^{k}\left|a_{x}-c_{x}\right|\right]\left[\Sigma_{x=1}^{\infty} x^{j-1}\left|\varphi_{g}(x)\right|\right]}{1-\Sigma_{x=1}^{k}\left|a_{x}\right|}$

Proof. From Theorem 4.1 we have for $j=0,1$

$$
\begin{aligned}
& \delta_{j}(f, g)=\Sigma_{x=1}^{\infty} x^{j-1}\left|\varphi_{f}(x)-\varphi_{g}(x)\right|= \\
& \sum_{x=1}^{\infty} x^{j-1}\left|x\left(a_{x}-c_{x}\right)+b_{x}-d_{x}+\Sigma_{y=1}^{k}\left(a_{y} \varphi_{f}(x-y)-c_{y} \varphi_{g}(x-y)\right)\right| \leq \\
& \sum_{x=1}^{k} x^{j-1}\left|x\left(a_{x}-c_{x}\right)+b_{x}-d_{x}\right|+\Sigma_{x=1}^{\infty} \sum_{y=1}^{k} x^{j-1}\left|a_{y}\right|\left|\varphi_{f}(x-y)-\varphi_{g}(x-y)\right|+ \\
& \sum_{x=1}^{\infty} \Sigma_{y=1}^{k} x^{j-1}\left|a_{y}-c_{y}\right|\left|\varphi_{g}(x-y)\right|= \\
& \Sigma_{x=1}^{k} x^{j-1}\left|x\left(a_{x}-c_{x}\right)+b_{x}-d_{x}\right|+\Sigma_{y=1}^{k}\left|a_{y}\right| \Sigma_{x=y+1}^{\infty} x^{j-1}\left|\varphi_{f}(x-y)-\varphi_{g}(x-y)\right|+ \\
& \sum_{y=1}^{k}\left|a_{y}-c_{y}\right| \Sigma_{x=y+1}^{\infty} x^{j-1} \mid \varphi_{g}^{(x-y) \mid \leq} \\
& \Sigma_{x=1}^{k} x^{j-1}\left|x\left(a_{x}-c_{x}\right)+b_{x}-d_{x}\right|+\Sigma_{y=1}^{k}\left|a_{y}\right| \Sigma_{x=y+1}^{\infty}(x-y)^{j-1}\left|\varphi_{f}(x-y)-\varphi_{g}(x-y)\right|+
\end{aligned}
$$


$\Sigma_{y=1}^{k}\left|a_{y}-c\right| \Sigma_{x=y+1}^{\infty}(x-y)^{j-1}\left|\varphi_{g}(x-y)\right|=\Sigma_{x=1}^{k} x^{j-1}\left|x\left(a_{x}-c_{x}\right)+b_{x}-d_{x}\right|+$ $\Sigma_{y=1}^{k}\left|a_{y}\right| \delta(f, g)+\Sigma_{y=1}^{k}\left|a_{y}-c_{y}\right| \Sigma_{x=1}^{\infty} x^{j-1}\left|\varphi_{g}(x)\right|$

from which we obtain (4.3).

Q.E.D.

We shall look at some special cases of Theorem 4.2. First we observe that when $\mathrm{c}=\mathrm{a}$, the last term in the numerator in (4.3) vanishes. Thus we obtain the following corollary.

Corollary 4.1. If the conditions of Theorem 4.2 are fulfilled with $\mathbf{c}=\mathbf{a}$, then

$\delta_{j}(f, g) \leq \frac{\Sigma_{x=1}^{k} x^{j-1}\left|b_{x}-d_{x}\right|}{1-\Sigma_{x=1}^{k}\left|a_{x}\right|}$.

If $k$ is large, then it might be interesting to use an approximation where we replace $a_{i}$ and $b_{i}$ with zero for $r<i \leq k$, that is, we replace the recursion (4.1) with the shorter recursion

$g(x)=\Sigma \underset{y=1}{r}\left[a_{y}+\frac{b}{x}\right] g(x-y) . \quad(x=1,2, \ldots)$

For such approximations we obtain the following corollary to Theorem 4.2.

Corollary 4.2. If the conditions of Theorem 4.2 are fulfilled with
${ }^{c}=a_{x} I(x \underline{r})$
$d_{x}=b_{x} I(\underline{x} r)$
$(x=1, \ldots, k)$ 
then

$$
\delta_{j}(f, g) \leq \frac{\Sigma_{x=r+1}^{k} x^{j-1}\left|x a_{x}+b_{x}\right|+\left[\Sigma_{x=r+1}^{k}\left|a_{x}\right|\right]\left[\Sigma_{x=1}^{\infty} x^{j-1}\left|\varphi_{g}(x)\right|\right]}{1-\Sigma_{x=1}^{k}\left|a_{x}\right|} .
$$

In the special case when $a_{x}=0$ for all $x>r$, such approximations become particularly attractive as then the last term in the numerator of (4.4) vanishes. For that case we obtain the following corollary.

Corollary 4.3. If the conditions of Corollary 4.2 are fulfilled with $a_{x}=0$ for $x=r+1, \ldots, k$, then

$\delta_{j}(f, g) \leq \frac{\Sigma_{x=r+1}^{k} x^{j-1}\left|b_{x}\right|}{1-\Sigma_{x=1}^{r}\left|a_{x}\right|}$.

In particular, it might be interesting to approximate the De Pril transform of $f$ by approximating $\varphi_{f}(x)$ with zero for $x>r$. In that case insertion in (3.1) gives immediately that the weak inequality (4.5) hold with equality. Thus we can state the following result.

Theorem 4.3. If $f \in \mathcal{P}_{0}$, and $g \in \mathcal{F}_{0}$ satisfies

$\varphi_{g}(x)=\varphi_{f}(x) I(x \underline{r})$

$(x=1,2, \ldots)$

then 
$\delta_{j}(f, g)=\Sigma \underset{x=r+1}{\infty} x^{j-1}\left|\varphi_{f}(x)\right| . \quad(j=0,1)$

From Theorem 4.3 we see that if $\nu_{j}(|f|)<w$, then $\delta_{j}(f, g)$ will go to zero if we let $r$ go to infinity, that is, we can obtain any desired degree of accuracy by choosing $r$ sufficiently large.

4B. In the special case when the function $g \in \mathcal{F}_{0}$ is in the form $R_{1}[a, b]$, Theorem 4.1 gives that

$\varphi_{g}(x)=(a+b) a^{x-1} . \quad(x=1,2, \ldots)$

Sundt \& Jewell (1981) proved the following theorem.

Theorem 4.4. The distribution $R_{1}[a, b]$ is binomial if $a<0$, Poisson if $a=0$, and negative binomial if $a>0$. In all these cases, we have $a+b \geq 0$.

Let us now apply the truncation scheme of Theorem 4.3 to the distribution $R_{1}[a, b]$. Then we get the following result.

Theorem 4.5. If $f$ is $R_{1}[a, b]$ with

$|a|<1$

and $g$ satisfies (4.6), then

$$
\delta_{1}(f, g)=(a+b) \frac{|a|^{r}}{1-|a|}
$$


$\delta_{0}(f, g)=\left|\frac{a+b}{a}\left[\ln (1-|a|)+\Sigma_{x=1}^{r} \frac{1}{x}|a|^{x}\right]\right| \leq \frac{\delta_{1}(f, g)}{r+1}$

Proof. Insertion of (4.8) in (4.7) gives

$\delta_{j}(f, g)=(a+b) \Sigma_{x=r+1}^{\infty} x^{j-1}|a|^{x-1}$,

from which we obtain

$$
\begin{aligned}
& \delta_{1}(f, g)=(a+b) \Sigma_{x=r+1}^{\infty}|a|^{x-1}=(a+b) \frac{|a|^{r}}{1-|a|} \\
& \delta_{0}(f, g)=(a+b) \Sigma_{x=r+1}^{\infty} \frac{1}{x}|a|^{x-1} \leq \frac{a+b}{r+1} \Sigma_{x=r+1}^{\infty}|a|^{x-1}=\frac{\delta_{1}(f, g)}{r+1} \\
& \delta_{0}(f, g)=\frac{a+b}{|a|}\left[\Sigma_{x=1}^{\infty} \frac{1}{x}|a|^{x}-\Sigma_{x=1}^{r} \frac{1}{x}|a|^{x}\right]= \\
& \left|\frac{a+b}{a}\left[\ln (1-|a|)+\Sigma_{x=1}^{r} \frac{1}{x}|a|^{x}\right]\right| .
\end{aligned}
$$

This completes the proof of Theorem 4.5.

Q.E.D.

We see that the bounds in Theorem 4.5 go to zero when $r$ goes to infinity, and thus we can obtain any desired degree of accuracy by choosing $r$ sufficiently large.

Let us apply Theorem 4.5 to the three cases in Theorem 4.4.

i) Binomial.

$f(x)=\left[\begin{array}{l}t \\ x\end{array}\right] \pi^{x}(1-\pi)^{t-x} . \quad(x=0,1 \ldots, t ; t=1,2, \ldots ; 0<\pi<1)$

Then

$$
a=-\frac{\pi}{1-\pi} \quad b=(t+1) \frac{\pi}{1-\pi}
$$


$\varphi_{f}(x)=-t\left[\frac{\pi}{\pi-1}\right]^{x}$

$(x=1,2, \ldots)$

To satisfy (4.9) we have to assume that $\pi<\frac{1}{2}$. In that case we obtain

$$
\delta_{0}(f, g)=t\left[\ln \frac{1-\pi}{1-2 \pi}-\Sigma_{x=1}^{r} \frac{1}{x}\left[\frac{\pi}{1-\pi}\right]^{x}\right] \quad \delta_{1}(f, g)=t \frac{\pi}{1-2 \pi}\left[\frac{\pi}{1-\pi}\right]^{r}
$$

ii) Poisson.

$f(x)=\frac{\lambda^{x}}{x !} e^{-\lambda}$

$(x=0,1, \ldots ; \lambda>0)$

Then

$$
\begin{array}{ll}
a=0 & b=\lambda \\
\varphi_{f}(x)=\lambda I(x=1) . & (x=1,2, \ldots)
\end{array}
$$

As $\varphi_{f}(x)=0$ for all $x>1$, the approximation is irrelevant.

iii) Negative binomial.

$f(x)=\left[\begin{array}{c}\alpha+x-1 \\ x\end{array}\right](1-\pi)^{\alpha} \pi^{x}$

$(x=0,1, \ldots ; \alpha>0 ; 0<\pi<1)$

Then

$$
\begin{array}{ll}
a=\pi & b=(\alpha-1) \pi \\
\varphi_{f}(x)=\alpha \pi^{x} . &
\end{array}
$$

As $|a|=\pi<1$, we can apply Theorem 4.5 , and we obtain 


$$
\delta_{0}(f, g)=\alpha\left|\ln (1-\pi)+\Sigma_{x=1}^{r} \frac{1}{x} \pi^{x}\right| \quad \delta_{1}(f, g)=\alpha \frac{\pi^{r+1}}{1-\pi}
$$

4C. Let us now discuss the possibility of extending Theorem 4.5 to the case when $f$ is $R_{k}[\mathbf{a}, \mathbf{b}]$ with $k<\infty$. As pointed out by Sundt (1993), in that case Theorem 4.1 gives a homogeneous linear difference equation of order $k$ with constant coefficients and $k$ constraints. The solution of this equation is in the form

$$
\varphi_{f}(x)=\Sigma{ }_{u=1}^{m} \Sigma_{v=1}^{t}{ }^{t} u v^{x^{v-1}} s_{u}^{x} \quad(x=1,2, \ldots)
$$

with $\Sigma_{u=1}^{m} t_{u}=k$ (cf. e.g. Henrici (1964, Corollary 6.8)). From Theorem 4.3 we obtain

$$
\delta_{j}(f, g)=\left.\Sigma_{u=1}^{m} \Sigma_{v=1}^{t} u c_{u v}\left|\Sigma_{x=r+1}^{\infty} x^{j+v-2}\right| s_{u}\right|^{x} . \quad(j=0,1)
$$

The upper bound in (4.13) is finite if and only if $\left|s_{u}\right|<1$ for $u=1, \ldots, m$, and in that case we can in principle proceed like in Theorem 4.5. However, it seems that the bound becomes rather weak unless $k$ is small. One should therefore reduce the order of the recursion if the distribution does not belong to $\mathcal{R}_{k}^{0}$. Furthermore, a distribution in $\mathcal{x}_{k}^{0}$ can often be represented as a convolution of distributions satisfying recursions of lower order, as a compound distribution with counting distribution in $\mathcal{R}_{l}$ with $k k$, or as a combination of these two cases. In the first case, we should combine Theorems 4.5 and 5.3, in the second case Theorems 4.5 and 6.3, and in the third case all three theorems. 


\section{Convolutions}

5A. When approximating $m$ distributions $f_{1}, \ldots, f_{m} \in \mathcal{P}_{0}$ by functions $g_{1}, \ldots, g_{m} \epsilon$ $\mathcal{F}_{0}$, which are not necessarily distributions themselves, it is also natural to approximate the convolution $f=*_{i=1}^{m} f_{i}$ by $g=*_{i=1}^{m} g_{i}$. To be able to do this, we have to extend the concept of convolutions to functions. We define the convolution $h_{1}^{*} h_{2}$ of two functions $h_{1}, h_{2} \in \mathcal{F}$ by

$$
\left(h_{1}^{*} h_{2}\right)(x)=\Sigma \underset{y=0}{x} h_{1}(y) h_{2}(x-y) . \quad(x=0,1, \ldots)
$$

Like for distributions, we define $h^{0^{*}}(x)=I(x=0)$ for a function $h \in \mathcal{F}$.

The following well-known properties of convolutions of distributions in $\mathcal{P}$ are easily extended to functions in $\mathcal{F}$ :

$$
\begin{aligned}
& h_{1}^{*} h_{2}=h_{2}{ }^{*} h_{1} \quad\left(h_{1}^{*} h_{2}\right)^{*} h_{3}=h_{1} *\left(h_{2}{ }^{*} h_{3}\right) \\
& h_{1}{ }^{*} h_{3}+h_{2}{ }^{*} h_{3}=\left(h_{1}+h_{2}\right)^{*} h_{3} .
\end{aligned}
$$

For a function $h \in \mathcal{F}$ we introduce the function $h \in \mathcal{F}$ defined by

$h(x)=x h(x)$.

$$
(x=0,1, \ldots)
$$

With this notation we can reformulate the relation (1.2) between a function $f \in \mathcal{F}_{0}$ and its De Pril transform as

$f=\varphi_{f}^{*} f$

This relation determines the De Pril transform uniquely. We also immediately see 
that

$\mu_{0}(\hbar)=\mu_{1}(h)$

Lemma 5.1. For $h_{1}, h_{2} \in \mathcal{F}$, we have

$\overline{h_{1}{ }^{*} h_{2}}=\bar{h}_{1}^{*} h_{2}+h_{1}^{*} h_{2}$.

Proof. For any non-negative integer $x$ we have

$\overline{h_{1}{ }^{*} h_{2}}(x)=x\left(h_{1}{ }^{*} h_{2}\right)(x)=x \Sigma{ }_{y=0}^{x} h_{1}(y) h_{2}(x-y)=\Sigma{ }_{y=0}^{x}[y+(x-y)] h_{1}(y) h_{2}(x-y)=$ $\Sigma_{y=0}^{x}\left[\hbar_{1}(y) h_{2}(x-y)+h_{1}(y) \hbar_{2}(x-y)\right]=\left(h_{1}^{*} h_{2}\right)(x)+\left(h_{1}^{*} h_{2}\right)(x)$,

which proves the lemma.

Q.E.D.

Theorem 5.1. If $h_{1}, h_{\mathscr{Z}} \in \mathcal{F}$ such that $\mu_{0}\left(\left|h_{i}\right|\right)<\infty$ for $i=1,2$, then

$\mu_{0}\left(h_{1}^{*} h_{2}\right)=\mu_{0}\left(h_{1}\right) \mu_{0}\left(h_{2}\right)$

If in addition $\mu_{1}\left(\left|h_{i}\right|\right)<\infty$ for $i=1,2$, then

$\mu_{1}\left(h_{1}^{*} h_{2}\right)=\mu_{0}\left(h_{1}\right) \mu_{1}\left(h_{2}\right)+\mu_{1}\left(h_{1}\right) \mu_{0}\left(h_{2}\right)$

If $h_{1}$ and $h_{2}$ are non-negative, then we can drop the conditions on $\mu_{j}\left(h_{i}\right)(i=1,2$; $j=0,1)$.

Proof. The first part of the theorem is proved by Dhaene \& Sundt (1994). 
If $\mu_{1}\left(\left|h_{i}\right|\right)<$ for $i=1,2$, then we obtain from Lemma 5.1 , the first part of the theorem, and (5.2)

$$
\begin{aligned}
& \mu_{1}\left(h_{1}^{*} h_{2}\right)=\mu_{0}\left(\overline{h_{1}{ }^{*} h_{2}}\right)=\mu_{0}\left(\bar{h}_{1}^{*} h_{2}+h_{1}^{*} h_{2}\right)=\mu_{0}\left(h_{1}^{*} h_{2}\right)+\mu_{0}\left(h_{1}^{*} \overline{h_{2}}\right)= \\
& \mu_{0}\left(h_{1}\right) \mu_{0}\left(h_{2}\right)+\mu_{0}\left(h_{1}\right) \mu_{0}\left(\overline{h_{2}}\right)=\mu_{1}\left(h_{1}\right) \mu_{0}\left(h_{2}\right)+\mu_{0}\left(h_{1}\right) \mu_{1}\left(h_{2}\right),
\end{aligned}
$$

which proves the second part of the theorem.

This completes the proof of Theorem 5.1.

Q.E.D.

We shall now extend Theorem 1.1 to De Pril transforms of functions in $\mathcal{F}_{0}$.

Theorem 5.2. The convolution of a finite number of functions in $\mathcal{F}_{0}$ is a function in $\mathcal{F}_{0}$, and its De Pril transform is the sum of the De Pril transforms of these functions.

Proof. Let $g_{1}, g_{\mathscr{Q}} \in \mathcal{F}_{0}$. Then

$$
\begin{aligned}
& \overline{g_{1}^{*} g_{2}}={\overline{g_{1}}}^{*} g_{2}+g_{1}{ }^{*} \overline{g_{2}}=\left(\varphi_{g_{1}}{ }^{*} g_{1}\right)^{*} g_{2}+g_{1}{ }^{*}\left(\varphi_{g_{2}}{ }^{*} g_{2}\right)= \\
& \varphi_{g_{1}}{ }^{*}\left(g_{1}{ }^{*} g_{2}\right)+\varphi_{g_{2}}{ }^{*}\left(g_{1}{ }^{*} g_{2}\right)=\left(\varphi_{g_{1}}+\varphi_{g_{2}}\right)^{*}\left(g_{1}{ }^{*} g_{2}\right),
\end{aligned}
$$

which implies that $\varphi_{g_{1}}{ }^{*} g_{2}=\varphi_{g_{1}}+\varphi_{g_{2}}$, that is, we have now proved that Theorem 5.2 holds for the convolution of two functions in $\mathcal{F}_{0}$. The general case follows easily by induction.

This completes the proof of Theorem 5.2.

Q.E.D.

From Theorem 5.2 we see that we can evaluate the approximation $g$ by first 
finding the De Pril transform of each $g_{i}$, then finding the De Pril transform of $g$ by by using Theorem 4.5, and finally finding $g$ recursively by (1.2).

The following theorem can be used together with the results in subsection $3 \mathrm{~A}$ to find error bounds for the approximation $g$.

Theorem 5.3. With $f_{i} \in \mathcal{P}_{0}$ and $g_{i} \in \mathcal{F}_{0}(i=1, \ldots, m)$, we have

$$
\begin{aligned}
& \delta\left(*_{i=1}^{m} f_{i}, *_{i=1}^{m} g_{i}\right) \leq \Sigma_{i=1}^{m} \delta\left(f_{i}, g_{i}\right) \\
& \delta_{j}\left(*_{i=1}^{m} f_{i}, *{ }_{i=1}^{m} g_{i}\right) \leq \Sigma_{i=1}^{m} \delta_{j}\left(f_{i}, g_{i}\right)
\end{aligned}
$$

and, if $\Sigma_{x=1}^{\infty}\left|\varphi_{f}(x)\right|<\infty$,

$\nu_{j}\left(* *_{i=1}^{m} g_{i}\right)=\Sigma_{i=1}^{m} \nu_{j}\left(g_{i}\right)$

Proof. For $j=0,1$, we have

$$
\begin{aligned}
& \delta_{j}\left(*_{i=1}^{m} f_{i}, *{ }_{i=1}^{m} g_{i}\right)=\Sigma_{x=1}^{\infty} x^{j-1}\left|\varphi_{f}(x)-\varphi_{g}(x)\right|= \\
& \Sigma_{x=1}^{\infty} x^{j-1}\left|\sum_{i=1}^{m}\left[\varphi_{f_{i}}(x)-\varphi_{g_{i}}(x)\right]\right| \leq \Sigma_{x=1}^{\infty} \Sigma_{i=1}^{m} x^{j-1}\left|\varphi_{f_{i}}(x)-\varphi_{g_{i}}(x)\right|= \\
& \Sigma_{i=1}^{m} \Sigma_{x=1}^{\infty} x^{j-1}\left|\varphi_{f_{i}}(x)-\varphi_{g_{i}}(x)\right|=\Sigma_{i=1}^{m} \delta_{j}\left(f_{i}, g_{i}\right) .
\end{aligned}
$$

Furthermore,

$$
\begin{aligned}
& \delta(f, g)=\delta_{0}(f, g)+\left|\ln \frac{f(0)}{g(0)}\right| \leq \Sigma_{i=1}^{m} \delta_{0}\left(f_{i}, g_{i}\right)+\left|\ln \prod_{i=1}^{m} \frac{f_{i}(0)}{g_{i}(0)}\right|= \\
& \Sigma_{i=1}^{m} \delta_{0}\left(f_{i}, g_{i}\right)+\left|\Sigma_{i=1}^{m} \ln \frac{f_{i}(0)}{g_{i}(0)}\right| \leq \Sigma_{i=1}^{m}\left[\delta_{0}\left(f_{i}, g_{i}\right)+\left|\ln \frac{f_{i}(0)}{g_{i}(0)}\right|\right]=\Sigma_{i=1}^{m} \delta\left(f_{i}, g_{i}\right) .
\end{aligned}
$$


If $\Sigma_{x=1}^{\infty}\left|\varphi_{f}(x)\right|<\infty$, then for $j=0,1$,

$$
\begin{aligned}
& \nu_{j}\left(*_{i=1}^{m} g_{i}\right)=\Sigma_{x=1}^{\infty} x^{j-1} \varphi_{g}(x)=\Sigma_{x=1}^{\infty} x^{j-1} \Sigma_{i=1}^{m} \varphi_{g_{i}}(x)= \\
& \Sigma_{i=1}^{m} \Sigma_{x=1}^{\infty} x^{j-1} \varphi_{g_{i}}(x)=\Sigma_{i=1}^{m} \nu_{j}\left(g_{i}\right) .
\end{aligned}
$$

This completes the proof of Theorem 5.3.

Q.E.D.

Theorem 5.3 shows that if we have upper bounds for the approximation error for the De Pril transform of each of the elements in the convolution, then we can easily find upper bounds for the approximation error for the De Pril transform of the convolution.

5B. By using the bounds of Theorem 5.3 in the bounds of Theorems 3.1 and 3.2 , we obtain

$$
\begin{aligned}
& \epsilon_{0}(f, g) \leq e^{\sum_{i=1}^{m} \delta\left(f_{i}, g_{i}\right)}-1 \\
& \eta(f, g) \leq\left[e^{\Sigma_{i=1}^{m} \delta\left(f_{i}, g_{i}\right)}-1\right]\left[\Sigma_{i=1}^{m} \mu_{1}\left(f_{i}\right)\right]+\Sigma_{i=1}^{m} \delta_{1}\left(f_{i}, g_{i}\right) e^{\sum_{i=1}^{m} \delta\left(f_{i}, g_{i}\right)} .
\end{aligned}
$$

The following theorem gives alternative bounds for $\epsilon_{0}(f, g)$ and $\eta(f, g)$.

Theorem 5.4. For $f_{i} \in \mathcal{P}$ and $g_{i} \in \mathcal{F}(i=1, \ldots, m)$, we have

$$
\begin{aligned}
& \epsilon_{0}\left(*_{i=1}^{m} f_{i}, *_{i=1}^{m} g_{i}\right) \leq \Sigma_{i=1}^{m}\left[\Pi_{j=i+1}^{m} \mu_{0}\left(\left|g_{j}\right|\right)\right] \epsilon_{0}\left(f_{i}, g_{i}\right) \leq \\
& \Sigma_{i=1}^{m}\left[\Pi_{j=i+1}^{m} \mu_{0}\left(\left|g_{j}\right|\right)\right]\left[e^{\delta\left(f_{i}, g_{i}\right)}-1\right] .
\end{aligned}
$$


If $f_{i}, g_{i} \in \mathcal{P}_{0}(i=1, \ldots, m)$, then

$\eta\left(*_{i=1}^{m} f_{i^{\prime}}, *_{i=1}^{m} g_{i}\right) \leq \Sigma_{i=1}^{m} \eta\left(f_{i^{\prime}}, g_{i}\right) \leq$

$\Sigma_{i=1}^{m}\left[\left[e^{\delta\left(f_{i}, g_{i}\right)}-1\right] \mu_{1}\left(f_{i}\right)+\delta_{1}\left(f_{i}, g_{i}\right) e^{\delta\left(f_{i}, g_{i}\right)}\right]$.

Proof. The first inequality in (5.5) and the first inequality in (5.6) were proved by Dhaene \& Sundt (1994), and the remaining inequalities follow by insertion of the upper bounds of Theorems 3.1 and 3.2.

This completes the proof of Theorem 5.4.

One somewhat disappointing aspect of (5.5) is that the bounds are not in general invariant against permutations of the pairs $\left(f_{i}, g_{i}\right)(i=1, \ldots, m)$.

We shall look at some special cases of Theorem 5.4.

Corollary 5.1. For $\in_{\in} \mathcal{P}_{0}, g \in \mathcal{F}_{0}$, and $m$ a positive integer, we have

$\epsilon_{0}\left(f^{m^{*}}, g^{m^{*}}\right) \leq \begin{cases}\frac{1-\mu_{0}(|g|)^{m}}{1-\mu_{0}(|g|)} \epsilon_{0}(f, g) \leq \frac{1-\mu_{0}(|g|)^{m}}{1-\mu_{0}(|g|)}\left[e^{\delta(f, g)}-1\right] & \left(\mu_{0}(|g|) \neq 1\right) \\ m \epsilon_{0}(f, g) \leq m\left[e^{\delta(f, g)}-1\right] . & \left(\mu_{0}(|g|)=1\right)\end{cases}$

If in addition $g \in \mathcal{P}_{0}$, then

$\eta\left(f^{m^{*}}, g^{m^{*}}\right) \leq m \eta(f, g) \leq m\left[\left[e^{\delta(f, g)}-1\right] \mu_{1}(f)+\delta_{1}(f, g) e^{\delta(f, g)}\right]$.

Corollary 5.2. For $f_{i} \in \mathcal{P}_{0}$ and $g_{i} \in \mathcal{F}_{0}$ with $\mu_{0}\left(\left|g_{i}\right|\right) \leq 1(i=1, \ldots, m)$, we have 
$\epsilon_{0}\left(*_{i=1}^{m} f_{i}, *_{i=1}^{m} g_{i}\right) \leq \Sigma_{i=1}^{m} \epsilon_{0}\left(f_{i}, g_{i}\right) \leq \Sigma_{i=1}^{m}\left[e^{\delta\left(f_{i}, g_{i}\right)}-1\right]$

The following theorem shows that under certain conditions Theorem 5.4 gives sharper bounds than (5.3) and (5.4).

Theorem 5.5. The bounds in (5.7) and (5.6) are sharper than the bounds in (5.3) and (5.4).

Proof. As

$\Sigma_{i=1}^{m}\left[e^{\delta\left(f_{i}, g_{i}\right)}-1\right] \leq \Pi_{i=1}^{m}\left[\left[e^{\delta\left(f_{i}, g_{i}\right)}-1\right]+1\right]-1=e^{\sum_{i=1}^{m} \delta\left(f_{i}, g_{i}\right)}-1$

we see that the bounds in (5.7) are sharper than the bound in (5.3). Furthermore,

$$
\begin{aligned}
& \sum_{i=1}^{m}\left[\left[e^{\delta\left(f_{i}, g_{i}\right)}-1\right] \mu_{1}\left(f_{i}\right)+\delta_{1}\left(f_{i}, g_{i}\right) e^{\delta\left(f_{i}, g_{i}\right)}\right] \leq \\
& \sum_{i=1}^{m}\left[\left[e^{\delta\left(f_{i}, g_{i}\right)}-1\right]\left[\Sigma_{r=1}^{m} \mu_{1}\left(f_{r}\right)\right]+\delta_{1}\left(f_{i}, g_{i}\right) e^{\delta\left(f_{i}, g_{i}\right)}\right] \leq \\
& {\left[e^{\Sigma_{i=1}^{m} \delta\left(f_{i}, g_{i}\right)}-1\right]\left[\Sigma_{i=1}^{m} \mu_{1}\left(f_{i}\right)\right]+\Sigma_{i=1}^{m} \delta_{1}\left(f_{i}, g_{i}\right) e^{\sum_{i=1}^{m} \delta\left(f_{i}, g_{i}\right)}}
\end{aligned}
$$

which shows that the bounds in (5.6) are sharper than the bound in (5.4).

This completes the proof of Theorem 5.5.

Q.E.D.

\section{Compound distributions}

6A. In this section we shall discuss approximations to compound 
distributions with counting distribution in $\mathcal{P}_{0}$ and severity distribution in $\mathcal{P}_{+}$.

Inspired by Gurland (1957), we shall denote the compound distribution with counting distribution $p \in \mathcal{P}_{0}$ and severity distribution $h \in \mathcal{P}_{+}$by $p \vee h$, that is,

$(p \vee h)(x)=\Sigma_{n=0}^{x} p(n) h^{n^{*}}(x), \quad(x=0,1, \ldots)$

and we extend this definition of the function $p \vee h$ to the case when $p \in \mathcal{F}_{0}$ and $h \in \mathcal{F}_{+}$. As

$(p \vee h)(0)=p(0)$

we see that $p \vee h \in \mathcal{F}_{0}$ when $p \in \mathcal{F}_{0}$.

To find a relation between the De Pril transforms of $p \vee h$ and $p$, the following lemma will be useful.

Lemma 6.1. For $h \in \mathcal{F}$, we have

$\overline{h^{n^{*}}}=\frac{n}{m} \overline{h^{m^{*}} * h^{(n-m)^{*}}}$.

$(m=1,2, \ldots, n ; n=1,2, \ldots)$

Proof. We shall first prove the lemma in the special case $m=1$, that is,

$\overline{h^{n^{*}}}=n \bar{h}^{*} h^{(n-1)^{*}}$

$(n=1,2, \ldots)$

by induction.

For $n=1,(6.2)$ trivially holds.

Let us now assume that (6.2) holds for $n=k$. Then, by Lemma 5.1, 
$\overline{h^{(k+1)^{*}}}=\bar{h}^{*} h^{k^{*}}+h^{*} h^{k^{*}}=\hbar^{*} h^{k^{*}}+h^{*}\left(k \bar{h}^{*} h^{(k-1)^{*}}\right)=\bar{h}^{*} h^{k^{*}}+k \bar{h}^{*} h^{k^{*}}=$ $(k+1) \hbar^{*} h^{k^{*}}$,

that is, (6.2) holds also for $n=k+1$, and by induction (6.2) holds for all integers $n$.

For positive integers $m$ and $n$ such that $m \leq n,(6.2)$ gives

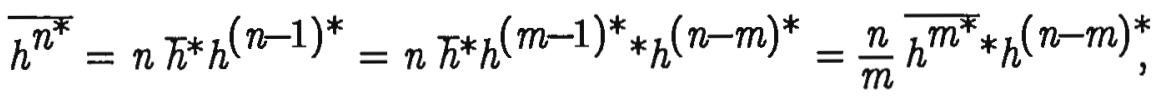

which proves the lemma.

Q.E.D.

Sundt (1993) deduced the following relation between the De Pril transforms of $p \vee h$ and $p$ in the case when $p \in \mathcal{P}_{0}$ and $h \in \mathcal{P}_{+}$.

Theorem 6.1. If $p \in \mathcal{F}_{0}$ and $h \in \mathcal{F}_{+}$, then

$\varphi_{p \vee h}(x)=x \Sigma_{y=1}^{x} \frac{\varphi_{p}(y)}{y} h^{y^{*}}(x) . \quad \quad(x=0,1, \ldots)$

Proof. For $x=0$ (6.3) trivially holds.

For any positive integer $x$ we have

$$
\begin{aligned}
& \overline{p \vee h}(x)=\Sigma_{n=1}^{x} p(n) \overline{h^{n^{*}}}(x)=\Sigma_{n=1}^{x} \frac{\left(\varphi_{p}^{*} p\right)(n)}{n} \overline{h^{n^{*}}}(x)= \\
& \Sigma_{n=1}^{x} \sum_{y=1}^{n} \frac{\varphi_{p}(y) p(n-y)}{n} \overline{h^{n^{*}}}(x)=\Sigma_{n=1}^{x} \Sigma_{y=1}^{n} \frac{\varphi_{p}(y) p(n-y)}{y} \overline{\left(h^{y^{*}}{ }_{h}(n-y)^{*}\right)(x)=} \\
& \Sigma_{y=1}^{x} \frac{\varphi_{p}(y)}{y} \Sigma_{n=y}^{x} p(n-y) \Sigma_{r=1}^{x} \overline{h^{y^{*}}}(r) h^{(n-y)^{*}}(x-r)= \\
& \Sigma_{r=1}^{x} \Sigma_{y=1}^{x} \frac{\varphi_{p}(y)}{y} \overline{h^{y^{*}}}(r) \Sigma_{n=y}^{x} p(n-y) h^{(n-y)^{*}}(x-r)= \\
& \Sigma_{r=1}^{x} \Sigma_{y=1}^{r} \frac{\varphi_{p}(y)}{y} \overline{h^{y^{*}}}(r) \Sigma_{m=0}^{x-y} p(m) h^{m^{*}}(x-r)=
\end{aligned}
$$




$$
\begin{aligned}
& \sum_{r=1}^{x} \sum_{y=1}^{r} \frac{\varphi_{p}(y)}{y} h^{y^{*}}(r)(p \vee h)(x-r)=\Sigma_{r=1}^{x}\left[\Sigma_{y=1}^{\infty} \frac{\varphi_{p}(y)}{y} \overline{h^{y^{*}}}\right](r)(p \vee h)(x-r)= \\
& {\left[\left[\sum_{y=1}^{\infty} \frac{\varphi_{p}(y)}{y} h^{y^{*}}\right] *(p \vee h)\right](x),}
\end{aligned}
$$

which by (5.1) implies that (6.3) holds for all positive integers $x$.

This completes the proof of Theorem 6.1.

Q.E.D.

The following theorem can sometimes be applied together with Theorem 3.4 to calculate $\mu_{0}(p \vee h)$ and $\mu_{1}(p \vee h)$.

Theorem 6.2. Let $p \in \mathcal{F}_{0}$ satisfying $\Sigma_{x=1}^{\infty}\left|\varphi_{p}(x)\right|<$ w and let $h \in \mathcal{F}+$ satisfying $\mu_{0}(h)=1$ and $\mu_{1}(|h|)<\infty$. Then

$$
\nu_{0}(p \vee h)=\nu_{0}(p) \quad \nu_{1}(p \vee h)=\nu_{1}(p) \mu_{1}(h)
$$

Proof. For $j=0,1$, we have

$$
\begin{aligned}
& \nu_{j}(p \vee h)=\Sigma_{x=1}^{\infty} x^{j-1} \varphi_{f}(x)=\Sigma_{x=1}^{\infty} x^{j} \Sigma_{y=1}^{\infty} \frac{\varphi_{p}(y)}{y} h^{y^{*}}(x)= \\
& \Sigma_{y=1}^{\infty} \frac{\varphi_{p}(y)}{y} \Sigma_{x=1}^{\infty} x^{j} h^{y^{*}}(x)=\Sigma_{y=1}^{\infty} y^{j-1} \varphi_{p}(y) \mu_{j}(h)=\nu_{j}(p) \mu_{j}(h),
\end{aligned}
$$

from which the theorem follows.

Q.E.D.

6B. One way to approximate a compound distribution, is to keep the severity distribution, but approximate the counting distribution. The following theorem is useful for finding bounds for the approximation errors of such approximations.

Theorem 6.3. Let $p \in \mathcal{P}_{0}, q \in \mathcal{F}_{0}$, and $h \in \mathcal{P}_{+}$. Then 


$$
\begin{aligned}
& \delta_{0}(p \vee h, q \vee h) \leq \delta_{0}(p, q) \\
& \delta_{1}(p \vee h, q \vee h) \leq \delta_{1}(p, q) \mu_{1}(h) \\
& \delta(p \vee h, q \vee h) \leq \delta(p, q) .
\end{aligned}
$$

Proof. By using Theorem 6.1 we obtain for $j=0,1$

$$
\begin{aligned}
& \delta_{j}(p \vee h, q \vee h)=\Sigma_{x=1}^{\infty} x^{j-1}\left|\varphi_{p \vee h}(x)-\varphi_{q \vee h}(x)\right|= \\
& \Sigma_{x=1}^{\infty} x^{j-1}\left|x \Sigma_{y=1}^{\infty} \frac{1}{y}\left(\varphi_{p}(y)-\varphi_{q}(y)\right) h^{y^{*}}(x)\right| \leq \\
& \Sigma_{x=1}^{\infty} x^{j} \Sigma_{y=1}^{\infty} \frac{1}{y}\left|\varphi_{p}(y)-\varphi_{q}(y)\right| h^{y^{*}}(x)=\Sigma_{y=1}^{\infty} \frac{1}{y}\left|\varphi_{p}(y)-\varphi_{q}(y)\right| \Sigma_{x=1}^{\infty} x^{j} h^{y^{*}}(x)= \\
& \Sigma_{y=1}^{\infty} y^{j-1}\left|\varphi_{p}(y)-\varphi_{q}(y)\right| \mu_{j}(h)=\delta_{j}(p, q) \mu_{j}(h),
\end{aligned}
$$

which proves (6.4) and (6.5). Formula (6.6) follows from (6.1) and (6.4).

This completes the proof of Theorem 6.3.

Q.E.D.

We see that if $\mu_{1}(h)<\infty$ and we let $q$ approach $p$ in such a way that $\delta_{0}(p, q)$ and $\delta_{1}(p, q)$ go to zero, then also $\delta_{0}(p \vee h, q \vee h)$ and $\delta_{1}(p \vee h, q \vee h)$ will go to zero.

The following theorem is obtained by application of Theorems 3.1, 3.2, 3.4, 6.2 , and 6.3 .

Theorem 6.4. If $p \in \mathcal{P}_{0}, q \in \mathcal{F}_{0}$, and $h \in \mathcal{P}_{+}$, then

$$
\begin{aligned}
& \epsilon_{0}(p \vee h, q \vee h) \leq e^{\delta(p, q)}-1 \\
& \eta(p \vee h, q \vee h) \leq\left[\left[e^{\delta(p, q)}-1\right] \mu_{1}(p)+\delta_{1}(p, q) e^{\delta(p, q)}\right] \mu_{1}(h) .
\end{aligned}
$$

5C. Whereas we in subsection $6 \mathrm{~B}$ discussed approximating compound distributions by approximating the counting distribution and keeping the severity distribution unchanged, we shall now consider the case when we approximate the severi- 
ty distribution and keep the counting distribution unchanged. For this situation Dhaene \& Sundt (1994) proved the following theorem.

Theorem 6.5. For $p \in \mathcal{F}$ and $h, k \in \mathcal{F}_{+}$with $\mu_{0}(|h|)<1$ and $\mu_{0}(|k|)<1$, we have

$\epsilon_{0}(p \vee h, p \vee k) \leq \mu_{1}(|p|) \epsilon_{0}(h, k)$

If in addition $h, k \in \mathcal{P}_{+}$, then

$\eta(p \vee h, p \vee k) \leq \mu_{1}(|p|) \eta(h, k)$

6D. We shall briefly mention two classes of approximations that can be convenient both for the counting distribution and the severity distribution in a compound distribution; for further details we refer to Dhaene \& Sundt (1994).

For $f \in \mathcal{P}$ we define the approximation $f^{(r)}$ for a positive integer $r$ by

$$
f^{(r)}(x)=f(x) I(x \leq r) . \quad(x=0,1,2, \ldots)
$$

For this approximation we have

$$
\begin{aligned}
& \epsilon_{0}\left(f, f^{(r)}\right)=1-\Gamma_{f}(r) \\
& \eta\left(f, f^{(r)}\right)=\epsilon_{1}\left(f, f^{(r)}\right)=\Pi_{f}(0)-\Pi_{f}(r)^{(0)}=\mu_{1}(f)-\mu_{1}\left(f^{(r)}\right)=\Pi_{f}(r)+r\left(1-\Gamma_{f}(r)\right) .
\end{aligned}
$$

We see that unless $\Gamma_{f}(r)=1$, the approximation $f^{(r)}$ will not be a distribution as $\mu_{0}\left(f^{(r)}\right)<\mu_{0}(f)=1$. To obtain a proper distribution, we can apply the modified approximation $f^{(r)}$ defined by 
$f^{(r)}(x)=\left\{\begin{array}{l}f(x) \\ 1-\Gamma f^{(r-1)} \\ 0 .\end{array}\right.$

$(x=0,1, \ldots, r-1)$
$(x=r)$
$(x=r+1, r+2, \ldots)$

We obtain

$\epsilon_{j}\left(f_{,} f^{(r)}\right)=\epsilon_{j}\left(f, f^{(r)}\right)+r^{j}\left(1-\Gamma_{f}(r)\right)$

$\eta\left(f, f^{(r)}\right)=\Pi_{f}(r)$

and notice that

$\epsilon_{j}\left(f_{,} \mathcal{F}^{(r)}\right) \geq \epsilon_{j}\left(f, f^{(r)}\right)$

$\eta\left(f, f^{(r)}\right) \leq \eta\left(f, f^{(r)}\right)$.

\section{Infinitely divisible distributions}

7A. A probability distribution $f \in \mathcal{P}$ is called infinitely divisible if there for each positive integer $m$ exists a distribution $f_{m} \in \mathcal{P}$ such that $f=f_{m}^{m^{*}}$. Let $\mathcal{P}^{\mathbf{m}}$ be the class of infinitely divisible distributions in $\mathcal{P}$, and let $\mathcal{P}_{0}^{\mathfrak{m}}=\mathcal{P} 0 \cap \mathcal{P}^{\mathfrak{m}}$. We see that if $f \in \mathcal{P}_{0}^{\mathfrak{m}}$, then $f_{m} \in \mathcal{P}_{0}^{\omega}$ for all integers $m$.

The following characterisation of distributions in $\mathcal{P}_{0}^{\infty}$ was given by Katti (1967) and, in terms of De Pril transforms, by Sundt (1994).

Theorem 7.1. A distribution in $\mathcal{P}_{0}$ is infinitely divisible if and only if its De Pril transform is non-negative.

Let $\mathcal{F}_{0}^{\infty}$ denote the class of functions in $\mathcal{F}_{0}$ with a non-negative De Pril trans- 
form. When approximating a distribution in $\mathcal{P}_{0}^{\infty}$, we shall often apply an approximation in $\mathcal{F}_{0}^{\infty}$. This is in particular the case with the truncation scheme of Theorem 4.3. The following lemma, which follows easily from (1.2) by induction, implies that such approximations will give a non-negative approximation to the distribution.

Lemma 7.1. All functions in $\mathcal{F}_{0}^{\infty}$ are non-negative.

The following theorem gives upper bounds for approximation errors related to approximations to infinitely divisible distributions.

Theorem 7.2. Let $f \in \mathcal{P}_{0}^{\infty}$ and $g \in \mathcal{F}_{0}^{\infty}$. If $\mu_{0}(g)<1$, then

$\epsilon_{0}(f, g) \leq \frac{1-\mu_{0}(g)}{\prod \ln \mu_{0}(g) \top} \delta(f, g)<\delta(f, g)$

If $g \in \mathcal{P}_{0}^{\infty}$, then

$\epsilon_{0}(f, g) \leq \delta(f, g)$

$\eta(f, g) \leq \delta_{1}(f, g)$.

Proof. As $f \in \mathcal{P}_{0}^{\infty}$, for each positive integer $m$ there exists a distribution $f_{m} \in \mathcal{P}_{0}^{\infty}$ such that $f=f_{m}^{m^{*}}$. From Theorem 1.1 follows that $\varphi_{f_{m}}=\frac{1}{m} \varphi_{f}$. Correspondingly we define $g_{m} \in \mathcal{F}_{0}^{\mathrm{w}}$ by $g_{m}(0)=g(0)^{\frac{1}{m}}$ and $\varphi_{g_{m}}=\frac{1}{m} \varphi_{g}$. Then $g=g_{m}^{m^{*}}$. We easily obtain

$\delta\left(f_{m}, g_{m}\right)=\frac{1}{m} \delta(f, g) \quad \delta_{1}\left(f_{m}, g_{m}\right)=\frac{1}{m} \delta_{1}(f, g)$ 
$\mu_{0}\left(g_{m}\right)=\mu_{0}(g)^{\frac{1}{m}}$

Let us now assume that $g \in \mathcal{P}_{0}^{\mathbb{w}}$. Then also $g_{m} \in \mathcal{P}_{0}^{\mathbb{w}}$, and from Corollary 5.1 and (7.4) we obtain

$\epsilon_{0}(f, g) \leq m\left[e^{\frac{1}{m} \delta(f, g)}-1\right] \quad \eta(f, g) \leq\left[e^{\frac{1}{m} \delta(f, g)}-1\right] \mu_{1}(f)+\delta_{1}(f, g) e^{\frac{1}{m} \delta(f, g)}$.

By letting $m$ go to infinity we obtain (7.2) and (7.3).

We now assume that $\mu_{0}(g)<1$. From (7.5) we see that also $\mu_{0}\left(g_{m}\right)<1$, and from Corollary 5.1 and (7.4) we obtain

$\epsilon_{0}(f, g) \leq \frac{1-\mu_{0}(g)}{1-\mu_{0}(g)^{\frac{1}{m}}}\left[e^{\frac{1}{m} \delta(f, g)}-1\right]$.

Letting $m$ go to infinity gives (7.1).

This completes the proof of Theorem 7.2.

Q.E.D.

We easily see that the bounds in (7.1)-(7.3) are sharper than the bounds in (3.2) and (3.3).

7B. If we approximate $f \in \mathcal{P}_{0}^{\mathrm{m}}$ by using the truncation scheme of Theorem 4.3, then the De Pril transform of the approximation $g$ will satisfy the inequalities

$0 \leq \varphi_{g}(x) \leq \varphi_{f}(x)$

$$
(x=1,2, \ldots)
$$


More generally, let $g \in \mathcal{F}_{0}^{\infty}$ be an approximation to $f$ that satisfies the inequalities (7.6) and

$0<g(0) \leq f(0)$

Then, by using induction on the recursion (1.2), we obtain that

$0 \leq g(x) \leq f(x)$

$(x=0,1, \ldots)$

This again implies that for $x=0,1, \ldots$,

$0 \leq \Gamma_{g}(x) \leq \Gamma_{f}(x)$

$0 \leq \Pi_{g}(x) \leq \Pi_{f}(x)$

$0 \leq \Pi_{g}^{(f)}(x) \leq \Pi_{f}(x)$.

In particular, (7.9) gives that

$0 \leq \mu_{0}(g) \leq \mu_{0}(f)=1$

and thus Theorem 7.2 applies for the present approximation $g$.

If $g$ is defined by (4.6) and $g(0)=f(0)$, then we have the nice relation

$\delta_{j}(f, g)=\nu_{j}(f)-\nu_{j}(g)$

$(j=0,1)$

If the inequalities in both (7.6) and (7.7) go the opposite way, then we also obtain the opposite inequalities in $(7.8)-(7.11)$. 
7C. For a proof of the following characterisation of infinitely divisible distributions, we refer to Feller (1968, Section XI.2).

Theorem 7.3. A distribution in $\mathcal{P}_{0}$ is infinitely divisible if and only if it can be expressed as a compound Poisson distribution with severity distribution on the positive integers.

Theorem 7.3 implies that if $f \in \mathcal{P}_{0}^{\infty}$, then there exists a distribution $h \in \mathcal{P}{ }_{+}$such that $f=p \vee h$ with

$p(n)=\frac{\lambda^{n}}{n !} e^{-\lambda}$

$$
(n=0,1, \ldots)
$$

and $\lambda$ determined by (6.1) and (7.12), that is,

$\lambda=|\ln f(0)|$.

Insertion of (4.11) in (6.3) gives

$\varphi_{f}(x)=x \lambda h(x)$

$(x=1,2, \ldots)$.

from which we obtain

$h(x)=\frac{\varphi_{f}(x)}{x \lambda}$

$(x=1,2, \ldots)$

Lemma 7.2. Let $g \in \mathcal{F}_{0}$, and let $p$ be the Poisson distribution given by (7.12). Then $g$ can be represented as $q \vee k$ with 


$$
\begin{array}{ll}
q(n)=\frac{g(0)}{p(0)} p(n) . & (n=0,1,2, \ldots) \\
k(x)=\frac{\varphi_{g}(x)}{x \lambda} . & (x=1,2, \ldots)
\end{array}
$$

Proof. As $q$ is equal to $p$ up to a multiplicative constant, $\varphi_{q}=\varphi_{p}$, and application of Theorem 6.1 and (4.11) gives for $x=0,1,2, \ldots$

$\varphi_{q \vee k}(x)=x \Sigma_{y=1}^{x} \frac{\varphi_{q}(y)}{y} k^{y^{*}}(x)=x \Sigma_{y=1}^{x} \frac{\varphi_{p}(y)}{y} k^{y^{*}}(x)=x \lambda k(x)=\varphi_{g}(x)$

Thus $q \vee k$ is equal to to $g$ up to a multiplicative constant, and as $(q \vee k)(0)=q(0)=$ $g(0)$, we see that $q \vee k=g$.

Q.E.D.

Theorem 7.4. If $f \in \mathcal{P}_{0}^{\infty}$ and $g \in \mathcal{F}_{0}$, then

$\delta_{j}(f, g)=\lambda \epsilon \hat{j}(h, k)$

with $\lambda$ given by (7.13), $h$ by (7.14), and $k$ by (7.15).

Proof. For $j=0,1$, we obtain

$\delta_{j}(f, g)=\Sigma_{x=1}^{\infty} x^{j-1}\left|\varphi_{f}(x)-\varphi_{g}(x)\right|=\lambda \Sigma_{x=1}^{\infty} x^{j}|h(x)-k(x)|=\lambda \epsilon_{j}(h, k) . \quad$ Q.E.D.

By combining Theorems 7.2 and 7.4 we obtain the following corollary.

Corollary 7.1. Let $f \in \mathcal{P}_{0}^{\infty}$ and $g \in \mathcal{F}_{0}^{\infty}$, and let $\lambda$ be given by (7.13), $h$ by (7.14), and $k$ by (7.15). If $\mu_{0}(g)<1$, then 
$\epsilon_{0}(f, g) \leq \frac{1-\mu_{0}(g)}{\prod \ln \mu_{0}(g) \mid}\left[\left|\ln \frac{f(0)}{g(0)}\right|+\lambda \epsilon_{0}(h, k)\right]<\left|\ln \frac{f(0)}{g(0)}\right|+\lambda \epsilon_{0}(h, k)$.

If $g \in \mathcal{P}_{0}^{\infty}$, then

$\epsilon_{0}(f, g) \leq\left|\ln \frac{f(0)}{g(0)}\right|+\lambda \epsilon_{0}(h, k) \quad \eta(f, g) \leq \lambda \epsilon_{1}(h, k)$

Let us consider Corollary 7.1 in the special case when $g(0)=f(0)$. In that case our approximation boils down to approximating $f=p \vee h$ by $g=p \vee k$. We see that the bounds in (7.17) and the weak bound in (7.16) are the same as the bounds obtained in Theorem 6.5.

We see that approximating $f$ by $g$ given by $g(0)=f(0)$ and

$\varphi_{g}(x)=\varphi_{f}(x) I(x \preceq r)$

$(x=1,2, \ldots)$

is equivalent with approximating $h$ by $h^{(r)}$ as defined in subsection 6D. Approximating $h$ by $\tilde{h}^{(r)}$ becomes equivalent with approximating $f$ by $g$ given by $g(0)=$ $f(0)$ and

$$
\varphi_{\tilde{g}}(x)= \begin{cases}\varphi_{f}(x) & (x=1,2, \ldots, r-1) \\ \varphi_{f}(r)+r \delta_{0}(f, g) & (x=r) \\ 0 . & (x=r+1, r+2, \ldots)\end{cases}
$$

As the approximation $\tilde{g}$ is obtained by replacing all severities greater than $r$ with $r$, we obtain the inequalities
$\Gamma_{\underset{g}{ }}(x) \geq \Gamma_{f}(x)$
$\Pi_{\tilde{g}}(x) \leq \Pi_{f}(x)$.
$(x=1,2, \ldots)$ 
An alternative way to modify the approximation $g$ so that the resulting approximation to $f$ becomes a probability distribution, is to rescale $g$, that is, apply the approximation

$\tilde{g}=\frac{1}{\mu_{0}(g)} g$.

We have $\varphi_{\tilde{g}}=\varphi_{g}$ and

$\delta_{j}(f, \tilde{g})=\delta_{j}(f, g)$

$\delta(f, \tilde{\tilde{g}})=\delta(f, g)-\ln \mu_{0}(g)$.

\section{Applications}

8A. In the preceding sections we have presented results about bounds for approximation errors in several simple situations. These results are building blocks that can be applied together in more complex situations.

For instance, let us consider a situation where we want to approximate the convolution $f$ of $m$ distributions $f_{1}, \ldots, f_{m}$. It is assumed that for each $i, f_{i}$ is a compound distribution $p_{i} V h_{i}$ with $p_{i} \in \mathcal{P}_{0}$ and $h_{i} \in \mathcal{P}_{+}$.

We approximate $f$ by for each $i$ approximating $p_{i}$ by a function $q_{i} \in \mathcal{F}_{0}$ obtained by truncating $\varphi_{p_{i}}$ at some positive integer $r$, that is,

$$
\varphi_{q_{i}}(x)=\varphi_{p_{i}}(x) I(x \underline{r} r) \quad(x=1,2, \ldots ; i=1,2, \ldots, m)
$$

with $q_{i}(0)=p_{i}(0)$. Hence, we approximate $f$ by $g=*_{i=1}^{m} g_{i}$ with $g_{i}=q_{i} \vee h_{i}$ 
From Theorem 4.3 we obtain that

$$
\delta_{j}\left(p_{i}, q_{i}\right)=\Sigma_{x=r+1}^{\infty} x^{j-1}\left|\varphi_{p_{i}}(x)\right| \quad(i=1,2, \ldots, m ; j=0,1)
$$

and from Theorem 6.3

$$
\begin{aligned}
& \delta\left(f_{i}, g_{i}\right)=\delta_{0}\left(f_{i}, g_{i}\right) \leq \delta_{0}\left(p_{i}, q_{i}\right)=\delta\left(p_{i}, q_{i}\right) \quad \delta_{1}\left(f_{i}, g_{i}\right) \leq \delta_{1}\left(p_{i}, q_{i}\right) \mu_{1}\left(h_{i}\right) . \\
& (i=1,2, \ldots, m)
\end{aligned}
$$

Application of Theorem 5.3 gives

$$
\begin{aligned}
& \delta(f, g) \leq \Sigma_{i=1}^{m} \delta\left(f_{i}, g_{i}\right) \leq \Sigma_{i=1}^{m} \delta\left(p_{i}, q_{i}\right) \\
& \delta_{1}(f, g) \leq \Sigma_{i=1}^{m} \delta_{1}\left(f_{i}, g_{i}\right) \leq \Sigma_{i=1}^{m} \delta_{1}\left(p_{i}, q_{i}\right) \mu_{1}\left(h_{i}\right) .
\end{aligned}
$$

Combining these bounds with the results in subsection $3 \mathrm{~A}$ gives error bounds for the approximation $g$ to $f$ and the corresponding approximations to $\Gamma_{f}$ and $\Pi_{f}$.

To evaluate $g$, we can for each $i$ evaluate $\varphi_{p_{i}}$ recursively by (1.1). From Theorem 6.1 we obtain

$\varphi_{g_{i}}(x)=x \Sigma{ }_{y=1}^{\infty} \frac{\varphi_{q_{i}}(y)}{y} h_{i}^{y^{*}}(x)=x \Sigma{ }_{y=1}^{r} \frac{\varphi_{p_{i}}(y)}{y} h_{i}^{y^{*}}(x) . \quad(x=1,2, \ldots ; i=1,2, \ldots, m)$

Application of Theorem 5.2 gives

$\varphi_{g}(x)=\Sigma{ }_{i=1}^{m} \varphi_{g_{i}}(x)=x \Sigma_{i=1}^{m} \Sigma_{y=1}^{r} \frac{\varphi_{p_{i}}(y)}{y} h_{i}^{y^{*}}(x)$ 
from which we can finally evaluate $g$ recursively by (1.2), starting with

$$
g(0)=f(0)=\Pi_{i=1}^{m} f_{i}(0)=\Pi_{i=1}^{m} p_{i}(0)
$$

8B. Let us now make the additional assumption that $p_{1}, \ldots, p_{m} \in \mathcal{P}_{0}^{\infty}$. Then the De Pril transforms of these discrete densities become non-negative, and thus we can drop the absolute value sign in (8.1).

As a compound distribution is infinitely divisible if its counting distribution is infinitely divisible, $f_{1}, \ldots, f_{m} \in \mathcal{P}_{0}^{\infty}$, and as a convolution of infinitely divisible distributions is infinitely divisible, $f \in \mathcal{P}_{0}^{\mathfrak{D}}$. It is easily shown that in the present case the weak inequalities in (8.2)-(8.4) become equalities.

From the discussion in subsection $7 \mathrm{~B}$ follows that

$0 \leq q_{i}(x) \leq p_{i}(x)$ $(x=0,1, \ldots ; i=1, \ldots, m)$

from which we obtain

$0 \leq g_{i}(x) \leq f_{i}(x)$ $(x=0,1, \ldots ; i=1, \ldots, m)$

This gives

$0 \leq g(x) \leq f(x)$ $(x=0,1, \ldots)$

from which we in particular obtain $\mu_{0}(g) \leq \mu_{0}(f)=1$ 
Hence Theorem 7.2 gives

$\epsilon_{0}(f, g) \leq \delta(f, g)$

To obtain bounds for approximations to stop loss premiums from Theorem 7.2 , we have to modify the approximation $g$ so that it becomes a probability distribution. One way to do this, is to for each $i$ modify $q_{i}$ so that it becomes a probability distribution, e.g. by using the modification given by (7.18).

8C. Instead of the assumption of infinite divisibility, we now assume that for each $i, p_{i}$ is $R_{1}\left[a_{i}, b_{i}\right]$ with $\left|a_{i}\right|<1$. From (4.8) we obtain that

$$
\varphi_{i}(x)=\left(a_{i}+b_{i}\right) a_{i}^{x-1} . \quad(x=1,2, \ldots)
$$

From Theorem 4.5 we obtain

$$
\begin{aligned}
& \delta_{0}\left(p_{i}, q_{i}\right)=\left|\frac{a_{i}+b_{i}}{a_{i}}\left[\ln \left(1-\left|a_{i}\right|\right)+\Sigma_{x=1}^{r} \frac{1}{x}\left|a_{i}\right|^{x}\right]\right| \leq \frac{a_{i}+b_{i}}{r+1} \frac{\left|a_{i}\right|^{r}}{1-\left|a_{i}\right|} \\
& \delta_{1}\left(p_{i}, q_{i}\right)=\left(a_{i}+b_{i}\right) \frac{\left|a_{i}\right|^{r}}{1-\left|a_{i}\right|^{\prime}} .
\end{aligned}
$$

By insertion of (8.5)-(8.7) in the expressions of subsection $8 \mathrm{~A}$, we obtain

$$
\begin{aligned}
& \varphi_{g}(x)=x \Sigma_{i=1}^{m}\left(a_{i}+b_{i}\right) \Sigma \underset{y=1}{r} \frac{a_{i}^{y-1}}{y} h_{i}^{y^{*}}(x) \quad(x=1,2, \ldots) \\
& \delta(f, g) \leq \Sigma_{i=1}^{m}\left|\frac{a_{i}+b_{i}}{a_{i}}\left[\ln \left(1-\left|a_{i}\right|\right)+\Sigma_{x=1}^{r} \frac{1}{x}\left|a_{i}\right|^{x}\right]\right| \leq \frac{1}{r+1} \Sigma_{i=1}^{m}\left(a_{i}+b_{i}\right) \frac{\left|a_{i}\right|^{r}}{1-\left|a_{i}\right|}
\end{aligned}
$$


$\delta_{1}(f, g) \leq \Sigma_{i=1}^{m}\left(a_{i}+b_{i}\right) \frac{\left|a_{i}\right|^{r}}{1-\left|a_{i}\right|^{r}} \mu_{1}\left(h_{i}\right)$.

We see that the upper bounds in (8.8) and (8.9) go to zero when $r$ goes to infinity. Thus we can obtain any desired degree of accuracy by choosing $r$ sufficiently large.

8D. De Pril (1989) considered the exact and approximate evaluation of the convolution $f$ of $m$ distributions $f_{1}, \ldots, f_{m} \in \mathcal{P}_{0}$. For each $i$ he represented $f_{i}$ as a compound Bernoulli distribution with counting distribution $p_{i}$ given by

$p_{i}(1)=1-p_{i}(0)=\pi_{i}=1-f_{i}(0)$

and severity distribution $h_{i} \in \mathcal{P}+$ given by

$h_{i}(x)=\frac{f_{i}(x)}{\pi_{i}}$.

$(x=1,2, \ldots)$

It is assumed that $\pi_{i}<\frac{1}{2}$.

As the Bernoulli distribution is in $x_{1}$, we can apply the results of the previous subsection. We have

$$
\begin{aligned}
& a_{i}=-\frac{\pi_{i}}{1-\pi_{i}} \quad b_{i}=2 \frac{\pi_{i}}{1-\pi_{i}} \\
& \varphi_{g}(x)=-x \Sigma_{y=1}^{r} \frac{1}{y} \Sigma_{i=1}^{m}\left[\frac{\pi_{i}}{\pi_{i}-1}\right]^{y} h_{i}^{y^{*}}(x) \quad(x=1,2, \ldots) \\
& \delta(f, g) \leq \Sigma_{i=1}^{m}\left[\ln \frac{1-\pi_{i}}{1-2 \pi_{i}}-\Sigma_{x=1}^{r} \frac{1}{x}\left[\frac{\pi_{i}}{1-\pi_{i}}\right]^{x}\right] \leq \frac{1}{r+1} \Sigma_{i=1}^{m} \frac{\pi_{i}}{1-2 \pi_{i}}\left[\frac{\pi_{i}}{1-\pi_{i}}\right]^{r}
\end{aligned}
$$


$\delta_{1}(f, g) \leq \Sigma_{i=1}^{m} \frac{\pi_{i}}{1-2 \pi_{i}}\left[\frac{\pi_{i}}{1-\pi_{i}}\right]^{r} \mu_{1}\left(h_{i}\right)$.

Together with the results in subsection $3 \mathrm{~A}$, the weak bound in (8.10) and the bound in (8.11) give the bounds found by De Pril (1989) and Dhaene \& De Pril (1994).

As we have seen above, De Pril's approximation can be considered as an approximation to the convolution of compound Bernoulli distributions. More generally, it can be considered as an approximation to the convolution of compound binomial distributions, as some of the distributions may be equal to each other. The set-up in subsection $8 \mathrm{C}$ generalises the set-up of De Pril in the sense that we allow some of the counting distributions to be negative binomial and Poisson as well as binomial.

8E. Let us now consider the case when all the counting distributions are negative binomial, that is, for $i=1, \ldots, m$

$p_{i}(n)=\left[\begin{array}{c}\alpha_{i}+n-1 \\ n\end{array}\right]\left(1-\pi_{i}\right)^{\alpha} \pi_{i}^{n}$

$\left(n=0,1, \ldots ; \alpha_{i}>0 ; 0<\pi_{i}<1\right)$

Then

$a_{i}=\pi_{i} \quad b_{i}=\left(\alpha_{i}-1\right) \pi_{i}$

Insertion in (8.5) gives

$\varphi_{p_{i}}(x)=\alpha_{i} \pi_{i}^{x} \geq 0$

$(x=1,2, \ldots)$ 
and from Theorem 7.1 follows that $p_{i}$ is infinitely divisible. Hence the discussion of subsection $8 \mathrm{~B}$ applies. In particular we obtain

$$
\begin{aligned}
& \varphi_{f}(x)=x \Sigma_{i=1}^{m} \alpha_{i} \Sigma_{y=1}^{\infty} \frac{\pi_{i}^{y}}{y} h_{i}^{y^{*}}(x) \\
& \varphi_{g}(x)=x \Sigma_{i=1}^{m} \alpha_{i} \Sigma_{y=1}^{r} \frac{\pi_{i}^{y}}{y} h_{i}^{y^{*}}(x) \quad(x=1,2, \ldots) \\
& \delta(f, g)=\Sigma_{i=1}^{m} \alpha_{i}\left|\ln \left(1-\pi_{i}\right)+\Sigma_{x=1}^{r} \frac{1}{x} \pi_{i}^{x}\right| \leq \frac{1}{r+1} \Sigma_{i=1}^{m} \alpha_{i} \frac{\pi_{i}^{r+1}}{1-\pi_{i}} \\
& \delta_{1}(f, g)=\Sigma_{i=1}^{m} \alpha_{i} \frac{\pi_{i}^{r+1}}{1-\pi_{i}} \mu_{1}\left(h_{i}\right) .
\end{aligned}
$$

\section{Acknowledgement}

The present research was carried out partly while the second author stayed at the Department of Mathematics at the Katholieke Universiteit Leuven with support from the Research Council of that university.

\section{References}

De Pril, N. (1989). The aggregate claims distribution in the individual model with arbitrary positive claims. ASTIN Bulletin 19, 9-24.

Dhaene, J. \& De Pril, N. (1994). On a class of approximative computation methods in the individual risk model. Insurance: Mathematics and Economics 14, 181-196.

Dhaene, J. \& Sundt, B. (1994). On error bounds for approximations to aggrge- 
gate claims distributions. In preparation.

Feller, W. (1968). An introduction to probability theory and its applications. Vol. 1 (3. ed.). Wiley, New York.

Gurland, J. (1957). Some interrelations among compound and generalized distributions. Biometrika 44, 265-268.

Henrici, P. (1964) Elements of numerical analysis. Wiley, New York.

Hipp, C. (1986). Improved approximations for the aggregate claims distribution in the individual model. ASTIN Bulletin 16, 89-100.

Katti, S.K. (1967). Infinite divisibility of integer-valued random variables. Annals of Mathematical Statistics 38, 1306-1308.

Kornya, P.S. (1983). Distribution of aggregate claims in the individual risk theory model. Transactions of the Society of Actuaries 35, 823-858.

Sundt, B. (1992). On some extensions of Panjer's class of counting distributions. ASTIN Bulletin 22, 61-80.

Sundt, B. (1994). On some properties of De Pril transforms of counting distributions. Submitted for publication in ASTIN Bulletin.

Sundt, B. \& Jewell, W.S. (1981). Further results on recursive evaluation of compound distributions. ASTIN Bulletin 13, 27-39.

Jan Dhaene

Departement Toegepaste Economische

Wetenschappen

Katholieke Universiteit Leuven

Naamsestraat 69

B-3000 Leuven

BELGIUM
Bjørn Sundt

The Wyatt Company A.S

P.O. Box 1508 Vika

$\mathrm{N}-0117$ Oslo

NORWAY 